\title{
URINARY TRACT INFECTIONS AND PRESSURE SORES AT GREEN PASTURES SPINAL INJURY UNIT
}

\author{
Herm F B*, Tripathi R B** Altrichter
}

\section{ABSTRACT}

Between 46-59\% of Spinal Cord Injury (SCI) cases develop Urinary Tract Infection (UTI) and 16-18\% develop pressure sores during the first year after the trauma. In a retrospective approach, the occurrence of UTI and pressure sores at Green Pastures Hospital's Spinal Injury Unit was studied. Between 1997-2000 out of 39 included patients 27 had suffered a thoracic-lumbar, 12 a cervical trauma. Twenty of these ( 51.2\% ) developed UTI and 7 ( $17.9 \%$ ) developed pressure sores during their hospitalization period. The most commonly organism found in both complications, UTI and pressure sores, was E. coli, followed by Proteus in UTI and Staph. aureus in pressure sores. Detected UTI and pressure sores among referred patients at admission was higher than expected $(71.7 \%$ and $48.7 \%$ respectively). In conclusion, adherence to a protocol by a multidisciplinary health care team resulted in favourable outcome of patients with SCI admitted to the rehabilitation unit. This is believed to be an indicator for the need of specialized SCI units. However, also in the studied sample at Green Pastures there was room for further improvement within the expected range.

\section{Key Words: Spinal Cord Injury, Urinary Tract Infection, Pressure Sore.}

\section{INTRODUCTION}

Urinary tract infections (UTI) and pressure sores (PS) are major complications and one of the main causes of morbidity in patients with spinal cord injuries (SCI). The incidence of UTI and PS is known to be closely related to the quality of bladder and skin care. ${ }^{1,2}$ However, also in specialized units in the West $46 \%$ of cases with SCI develop UTI and $18 \%$ pressure sores during the first 6 weeks after the SCI trauma. 59\% of these SCI cases develop UTI and $16 \%$ pressure sores within the following first year after trauma. ${ }^{1,2}$ In July 1997, the first spinal cord injury patients were admitted to the rehabilitation unit at Green Pastures Hospital and since then 10 - 20 SCI cases from the Western regions of Nepal have been treated in this specialized unit every year. Outcome studies have been reported recently. ${ }^{3,4}$ The present study's aim was to evaluate the quality of care provided in the specialized unit and therefore looked into the occurrence of UTI and pressure sores among the SCI cases admitted between 1997 and 2000.

\section{PATIENTS AND METHODS}

Spinal cord injury cases (SCI) were referred mainly from various hospitals in the Western region of Nepal, such as the Western Regional Hospital in Pokhara, the UMN hospitals in Tansen and Amp Pipal, the Manipal Teaching Hospital and other hospitals. Between July 1997 and August 2000 a total of 47 SCI cases were admitted to Green Pastures rehabilitation unit. The sample studied included 39 cases as eight of the patients were excluded due to lack of data on UTI and pressure

* Green Pastures Hospital, Pokhara, Nepal.

** Western Regional Hospital, Kaski, Nepal.

*** University of Halle / Saale, Germany.

Address for correspondence : $\quad$ Dr. Friedbert B. Herm

Green Pastures Hospital

P.O. Box: 28, Pokhara, Nepal

Email: gph@inf.org.np 
sores. Among the remaining 39 cases, 27 had suffered a thoracic-lumbar trauma and 12 a cervical trauma. ${ }^{5}$ Paraplegics comprised a group of complete and incomplete spinal cord lesions, while only incomplete cervical lesions were admitted to the unit according to the admission protocol. Male / female ratio was $2.25 / 1$, mean age 38.7 years and the average length of stay 122.9 days (table I). Clinical records and laboratory records were reviewed to determine the prevalence of urinary

new pressure sores during admission (table II and III). The most common organisms found in both complications, UTI and pressure sores, were E. coli, followed by Proteus in UTI and Staph. aureus in pressure sores (table IV). The main location of pressure sores was the sacrum $(39 \%)$ followed by the hip (34.1\%), heel / ankle region and other locations (i.e. Occipital). In 30 cases a urinary catheter was indicated because of the absence of normal bladder function. As well as Foley catheters (53.3\%), condom catheters $(26.7 \%)$, intermittent catheterization (16.6\%) and suprapubic catheterization were also used (table II). For the surgical management of pressure sores 37 interventions were performed: 26 septic and 11 plastic procedures (table III). SCI patients were discharged only after complete 